\title{
Intervención ambiental en sitios contaminados por plomo: la experiencia en los Estados Unidos de América
}

\author{
Nicholas Ceto, BS, MS. ${ }^{(1)}$
}

\begin{abstract}
Ceto N.
Intervención ambiental en sitios contaminados por plomo: la experiencia en los Estados Unidos de América. Salud Publica Mex 2003;45 supl 2:S232-S236. El texto completo en inglés de este artículo está disponible en: http://www.insp.mx/salud/index.html
\end{abstract}

\section{Resumen}

Durante una década, los organismos estatales y federales han trabajado de manera conjunta con las comunidades a lo largo del territorio estado unidense, con el objeto de valorar el riesgo a la salud que implica la contaminación por plomo en las zonas residenciales. A menudo dichas comunidades han estado vinculadas con instalaciones destinadas anteriormente a actividades como la minería y la metalurgia; sin embargo, existen otras industrias como por ejemplo la fabricación de pinturas y el reciclaje de baterías, también identificadas como fuentes de contaminación por este metal. La vasta experiencia en las tareas de limpieza de los sitios contaminados ha puesto de manifiesto que los amplios programas destinados a identificar y manejar las vías de exposición pueden ayudar, de manera efectiva, a disminuir los niveles de plomo en sangre de las poblaciones más suceptibles, como la de los de niños en edades tempranas. Los programas de intervención ambiental son más efectivos cuando en las localidades afectadas se ejecutan programas de educación/intervención en salud, orientados al desarrollo de estrategias individualizadas para manejar el riesgo que implica la presencia de dicho metal en el ambiente. El texto completo en inglés de este artículo está disponible en: http://www.insp.mx/salud/index.html

Palabras clave: plomo; contaminación ambiental; política ambiental; Estados U nidos

\author{
Ceto $\mathrm{N}$. \\ Environmental intervention in sites contaminated by lead: \\ The United States of America experience. \\ Salud Publica Mex 2003;45 suppl 2:S232-S236. \\ The English version of this paper \\ is available at: http://www.insp.mx/salud/index.html
}

\begin{abstract}
A bstract
For a decade, state and federal agencies have worked jointly with communities throughout the USA, with the objective of measuring the health-risk from lead pollution in residential zones. 0 ften these communities have been linked with facilities previously associated with activities like mining and metallurgy; nevertheless, there are other industries like paint manufacturing and battery recycling, that have also been identified as lead pollution sources. The vast experience in cleaning up the contaminated sites has shown that ample programs designed to identify and handle the exposure routes can help, in an effective manner, to diminish blood lead levels (BLL) in susceptible populations, such as in young children. Environmental inter vention programs are more effective when the affected communities carry out health education/intervention programs, geared towards the development of individualized strategies for handling the risk implied by the presence of lead in the atmosphere. The English version of this paper is available at: http:// www.insp.mx/salud/index.html
\end{abstract}

Key words: lead; environmental pollution; environmental policy; U nited States

(1) Universidad de Vermont, Seattle, W ashington, Estados Unidos de A mérica.

Fecha de recibido: 1 de febrero de 2002 - Fecha de aprobado: 8 de octubre de 2002

Solicitud de sobretiros: $N$ icholas Ceto. Coordinador Regional de Minería. A gencia Estadounidense para la Protección A mbiental, 1200 Sixth Avenue. Seattle,W ashington, Estados Unidos de América, 98100

Correo electrónico: ceto.nicholas@epa.gov 
D urante una década, los organismos estatales y federales han trabajado con las comunidades en los Estados Unidos de América (EUA) con el fin de evaluar y manejar los riesgos para la salud que implica la contaminación por plomo en las áreas residenciales. Gran parte de los trabajos se iniciaron a partir de la promulgación del Comprehensive Environmental Response Compensation and Liability Act (CERCLA o Superfund, por su siglas en inglés). A menudo las comunidades afectadas se vinculan con sitios e instalaciones en donde se practicaba la minería o bien la metalurgia; sin embargo, existen otras actividades industriales también identificadas como fuentes de contaminación: la fabricación de pintura y el reciclamiento de baterías.

La experiencia obtenida en los EUA demuestra que un programa multimedia, cuidadosamente concebido y puesto en marcha para evaluar las fuentes de contaminación por plomo, así como sus vías de acceso a los seres humanos, puede ayudar a mitigar la exposición y dar como resultado índices de exposición más bajos. Considerando que los niños que residen en una comunidad afectada son particularmente susceptibles a los efectos de esta exposición, sus niveles de plomo en sangre (NPS) son evaluados constantemente con el fin de contar con un indicador de la exposición en curso. Este documento aporta una visión general de las estrategias de intervención ambiental más frecuentemente utilizadas para mitigar la exposición a esta contaminación en un ámbito residencial. Se proporciona un ejemplo de este enfoque aplicado a un caso específico en los EUA.

\section{Desarrollo de las metas del proyecto}

Un primer paso crítico en el desarrollo de una estrategia de intervención ambiental constituye el establecimiento de metas claras. Estas, llamadas en ocasiones Objetivos de la Acción de Remediación (Remedial Action Objectives -RAOs, por sus siglas en inglés-) aportan una base para el desarrollo de los elementos esenciales para este programa, además de identificar las estrategias específicas de mitigación que permitirán evaluar las vías de exposición a este metal. A continuación se proporcionan algunos ejemplos de RAOs.

- Desarrollar estrategias para reducir el riesgo que evalúen todas las fuentes potenciales de exposición a plomo, incluyendo suelos, polvo, emisiones al aire, sistema de plomería, agua potable, pintura, productos para el hogar, exposición ocupacional secundaria, artesanías o pasatiempos, fuentes de alimentación y otros elementos específicos

- Limitar la exposición a los niveles de plomo en suelo a tal punto que cualquier niño, o que los niños, cuya exposición es similar, corran un riesgo estimado de no más de $5 \%$ de NPS superior a los $10 \mu \mathrm{g} / \mathrm{dl}$

- Enfocar las primeras acciones con las estrategias destinadas a aminorar el riesgo en poblaciones sensibles

- Diseñar estrategias confiables de intervención ambiental a lo largo del tiempo

- Diseñar e instaurar programas compatibles con las metas y las capacidades de la comunidad.

\section{Elementos de un programa de intervención ambiental}

Una vez establecidas las metas generales, los encargados de manejar el sitio contaminado pueden comenzar a trabajar con la comunidad afectada con el fin de identificar los elementos básicos de un programa de intervención ambiental con estas características. Es fundamental que la comunidad participe activamente en esta etapa del proceso, puesto que la efectividad de estos programas depende de la ejecución de los miembros de la comunidad, y deben contar con el apoyo de los programas del gobierno local.

En aquellos sitios donde son necesarios programas de largo plazo para aminorar los riesgos por exposición a este metal, es necesario contar con un programa amplio que valore los riesgos tanto de corto como de largo plazo. Este tipo de programas son los idóneos en aquellos sitios donde es imposible eliminar totalmente esta contaminación y donde es necesario un manejo a largo plazo para evitar una contaminación posterior debida a los medios ya contaminados. Los elementos fundamentales de un programa efectivo se caracterizan por incluir estrategias para:

- Reducir la exposición a los medios ambientalmente contaminados

- Educar de manera amplia a la comunidad respecto del riesgo que representa el plomo

- Proporcionar servicios de intervención en materia de salud a las poblaciones potencialmente expuestas

- Desarrollar una infraestructura comunitaria para proporcionar un apoyo a largo plazo a través de los elementos de programas de intervención ambiental

- Llevar a cabo monitoreos para evaluar la efectividad del programa, y mofidicarlo en caso necesario. 


\section{Estrategias para mitigar la exposición a plomo}

Resulta necesario desarrollar estrategias para mitigar la exposición, que permitan identificar y conocer las vías de exposición específicas de cada sitio. Si bien el desarrollo detallado de estrategias es particular a cada sitio, existen diversas estrategias de orden general comunes a los programas de intervención ambiental en los sitios de contaminación por plomo más complejos en los EUA. En estas estrategias se manejan objetivos de salud pública gracias a la puesta en marcha de programas de intervención ambiental multimedia, complementados con iniciativas orientadas a lograr una reducción temprana del riesgo.

- Control de las fuentes activas de emisión de plomo en la atmósfera, incluyendo tanto las fuentes fijas, como el polvo que se volatiliza. La instalación de equipos para controlar las emisiones en las fuentes fijas constituye un ejemplo

- Poner en marcha programas de educación e intervención en salud en un intento por limitar la exposición a plomo mientras queda bien afianzado el control de las fuentes de contaminación; es esencial la participación de los funcionarios de salud de la localidad, así como de la comunidad misma en el desarrollo y establecimiento del programa

- Los programas de educación prescolar que han fomentado que los niños se laven las manos antes de comer, limitando así la exposición a plomo, constituyen un ejemplo

- Concentrar los esfuerzos de control temprano de las fuentes en aquellas áreas donde se encuentren grandes cantidades de niños de corta edad potencialmente expuestos, incluyendo escuelas, campos de juego, iglesias, campos destinados a la práctica de deportes e instalaciones para el cuidado de los niños. El remplazo de los suelos contaminados en un campo de futbol constituye un ejemplo

- Una vez que las fuentes de exposición de las áreas de uso común han sido mitigadas será necesario enfocar los esfuerzos para controlar la exposición en los hogares habitados por subpoblaciones más sensibles a la misma. El remplazo de los suelos contaminados de los sitios donde residen niños menores de tres años de edad, constituye un ejemplo

- Las medidas para mitigar la exposición en el hogar deberán permitir el manejo de las fuentes específicas de exposición multimedia para cada sitio.
Un ejemplo es el remplazo de la tubería con soldadura de plomo, por otra que no la tenga

- En el diseño de las estrategias para manejar los riesgos que implica la exposición a suelos contaminados se deberá tomar en cuenta el suelo cercano a las vías de ingreso que sigue el polvo en los hogares. Para poder reducir, a largo plazo, la cantidad de polvo contaminado con plomo que penetra en las casas es necesario un proceso previo de limpieza que abarque a toda la comunidad. La limpieza a fondo del interior de las casas, una vez que se han remplazado los suelos contaminados, constituye un ejemplo

- Proporcionar equipo a los residentes con el fin de que sean ellos mismos quienes limpien sus casas puede llegar a ser una medida temporal efectiva para controlar la exposición al polvo dentro del hogar, en tanto se ponen en vigor las medidas necesarias para controlar la fuente de contaminación. Los programas que ofrecen aspiradoras, en préstamo, a los propietarios de las casas, constituyen un ejemplo

- Las estrategias para remediar la condición de los suelos pueden incluir la eliminación y el remplazo de aquellos contaminados, o bien repararlos, recubrirlos con una capa de vegetación o restringir su acceso. El recubrir con asfalto las entradas contaminadas para coche constituye un ejemplo

- Los controles ejercidos por medio de las instituciones pueden ser una parte integral de una estrategia general para manejar, a largo plazo, los suelos contaminados que se conserven in situ, como parte de un programa de limpieza basado en la (el) contención/recubrimiento y no en la (el) eliminación/tratamiento del material contaminado. El local zoning que requiere de una licencia para excavar los suelos contaminados, seguido de una correcta eliminación, constituye un ejemplo.

\section{Un estudio de caso: el sitio de Bunker Hill Superfund}

Cuando se diseñó una estrategia de limpieza a fondo para el sitio de Bunker Hill Superfund, ubicado en el norte de Idaho, se incluyeron muchos de los objetivos y estrategias ya descritos. Se trata de una área mixta, de carácter residencial, comercial e industrial, que se ha ido contaminando con metales, en particular plomo, liberados en el ambiente a lo largo de más de 100 años de actividades mineras y metalúrgicas. La comunidad de Kellog, en Idaho, es la más grande del área afectada. 
En 1974, y como resultado de una falla en el equipo de control de emisiones de la fundidora de plomo de Bunker Hill, el NPS promedio de los niños que se encontraban en el área inmediata a la fundidora era de casi $70 \mu \mathrm{g} / \mathrm{dl}$. Hoy en día, un NPS que rebasa los 10 $\mu \mathrm{g} / \mathrm{dl}$ es considerado como motivo de preocupación. Cuando las emisiones de la fundidora estuvieron nuevamente bajo control, los NPS disminuyeron, aunque siguieron siendo elevados, con un promedio de casi $40 \mu \mathrm{g} / \mathrm{dl}$ en la comunidad de Kellog, hasta que se cerró la fundidora en 1981. Para 1983 el promedio de los NPS de los niños de dicha comunidad había descendido aproximadamente a los $20 \mu \mathrm{g} / \mathrm{dl}$, cifra muy por debajo de los niveles históricos que, sin embargo, seguía siendo elevada debido a la vasta contaminación de que era objeto la comunidad. En 1983 comenzaron las tareas para tipificar las condiciones ambientales y evaluar y manejar los riesgos a la salud humana y al entorno; en esa fecha, Bunker Hill quedó incluido en la lista de los sitios más gravemente contaminados del país.

Las primeras acciones se orientaron al control del polvo que se dispersaba, así como a identificar las áreas de uso común contaminadas, donde las tareas de limpieza podrían tener el efecto más considerable. Asimismo, se puso en marcha un enérgico programa educativo y de intervención en salud con el fin de complementar las primeras acciones para controlar las fuentes de exposición. En el programa se manejaban todas las fuentes de exposición que pudieran existir al interior de las casas.

En 1989 arrancó un programa cuyo objetivo era eliminar y remplazar, o bien recubrir, los suelos de las casas contaminadas. Las casas habitadas por niños pequeños y por mujeres embarazadas fueron objeto de la más alta prioridad. A la fecha se ha intervenido en más de 1800 hogares. De manera gratuita se pusieron a la disposición de los residentes del área aspiradoras especiales para la limpieza de las casas, en un esfuerzo por minimizar el contacto con el polvo contaminado. Los pozos de agua contaminada fueron clausurados. Se establecieron acciones complementarias para manejar la contaminación en las áreas comerciales, así como en el antiguo complejo industrial, con el fin de controlar la exposición en esas áreas y limitar la contaminación de nueva cuenta de las áreas adyacentes, donde ya se habían llevado a término las acciones de limpieza. Por otra parte, se pusieron en marcha controles de carácter institucional, que incluían a toda la comunidad del sitio, para facilitar el manejo, a largo plazo.
Estas vastas acciones han tenido un notable efecto benéfico en los NPS de la comunidad: hoy en día el NPS promedio en los niños es de aproximadamente $5 \mu \mathrm{g} / \mathrm{dl}$, a diferencia de los $20 \mu \mathrm{g} / \mathrm{dl}$ registrados en 1983. Asimismo, las condiciones ambientales en el área circundante han mejorado de manera considerable. Se han obtenido logros similares en otros sitios contaminados en los EUA, donde se han ejecutado amplios programas para aminorar esta exposición. En 1995 en Jasper County, Missouri, un sitio contaminado por plomo, cerca de $14 \%$ de los niños con NPS excedían los $10 \mu \mathrm{g} / \mathrm{dl}$. En $1999 \mathrm{y}$ a partir de la puesta en marcha de un programa de educación/intervención en salud, así como de proyectos de intervención ambiental, la cifra bajó a $6 \%$.

\section{Lecciones aprendidas}

La experiencia de una década de acciones para limpiar sitios contaminados ha demostrado que los programas amplios para manejar las vías de exposición al plomo permiten disminuir los NPS en poblaciones que corren más riesgo, como los niños de poca edad. Los programas de intervención ambiental resultan más efectivos cuando son complementados por programas de intervención en salud y educación, aplicados en las localidades afectadas y cuyo objetivo es el desarrollo de estrategias particulares para manejar el riesgo que implica la presencia de este metal.

En la ejecución de dichos programas varias son las lecciones que pueden aplicarse en otros sitios en donde se enfrentan problemas similares. Los principios que se destacan son:

- La conciencia y la participación comunitarias son esenciales para el éxito del proyecto

- Es importante realizar un análisis multimedia/ multivía de la exposición al plomo, con el fin de diseñar estrategias efectivas para mitigar dicha exposición

- Los programas de educación e intervención en salud comunitarias constituyen un complemento esencial para aminorar las fuentes de exposición ambiental, y su diseño y puesta en marcha deberán ser altamente prioritarios

- En los lugares amplios a menudo será necesario establecer un manejo in situ del material que constituye una fuente de contaminación; en los sitios más reducidos la eliminación total de éste podrá resultar costo-efectiva puesto que los programas de educación/intervención, los controles insti- 
tucionales y el monitoreo pueden resultar innecesarios

- La posibilidad de que ocurra una nueva contaminación debe tomarse en cuenta si las actuales fuentes no han sido completamente controladas, o bien si la limpieza del sitio ha sido programada por un periodo prolongado

- Los controles institucionales (CI) a menudo son un componente necesario en la planeación del proyecto, para proteger la integridad de las medidas y aminorar la exposición

- Los monitoreos ambientales y de salud son esenciales para determinar si se han logrado las metas del proyecto.

El éxito en el manejo de los sitios contaminados por plomo puede alcanzarse si los encargados de aplicarlo trabajan de manera conjunta con las autoridades locales en la identificación de las metas adecuadas del proyecto, diseñando y ejecutando seguidamente estrategias de intervención ambiental y programas de intervención en salud y educación que respondan a dichas metas. Conviene esperar que las consideraciones relativas a la planeación y la puesta en marcha discutidos en este documento, resulten útiles en ese proceso.

\section{Lecturas adicionales para completar la información presentada en este documento}

United States Environmental Protection A gency. Guidance for coducting remedial investigations and feasibility studies under CERCLA-Interim final, 0 ffice of Emergency and Remedial Response.W ashington, DC: US EPA, 1988;EPA/540/G -89/004, O SW ER Directive 9355.3-01.

United States Environmental Protection A gency Region 10. Bunker hill mining and metallurgical complex record of decision. 1991;Seattle (W A):US/EPA, EPA/RO D/R10-91/028.

United States Environmental Protection A gency. Clarification to the 1994 revised interim soil lead guidance for CERCLA sites and RCRA corrective action facilities, 0 ffice of Solid W aste and Emergency Response.W ashington, D.C.: US EPA, 1998;EPA/540/F-98/030, O SW ER Directive 9200.4-27.

US Department of Health and Human Services. The nature and extent of lead poisoning in children in the U nited States:A report to Congress, A gency for Toxic Substances and D isease Registry, Public Health Service. Atlanta (GA): US DHHS; 1988.

United States Environmental Protection A gency Region 7. 0 ronogoDuenweg Mining Belt-Jasper County site record of decision, Kansas City (MO ): US/EPA, 1996;EPA/RO D/R07-96/086.

United States Environmental Protection A gency Region 10. Bunker Hill Populated A reas 0 perable Unit, first five year review report, Seatle (W A): USA/EPA, 2000. 